\title{
LES BASES MÉTHODOLOGIQUES D'UN PROJET DE GESTION DES POISSONS MIGRATEURS SUR LE BASSIN VERSANT DE LA DORDOGNE.
}

\author{
G. PUSTELNIK, O. GUERRI
}

\author{
E.P.I.DOR., Etablissement Public Interdépartemental DORdogne \\ B.P. 13, 24250 Castelnaud-la-Chapelle, France.
}

Reçu le 05 janvier 2000

Accepté le 21 septembre 2000
Received 05 January, 2000 Accepted 21 September, 2000

\section{RÉSUMÉ}

Les opérations de restauration des poissons migrateurs ont débuté en 1978 sur le bassin de la Dordogne. Vingt ans après, de nombreux acquis en matière de connaissances, de réglementations, d'aménagements physiques et d'interventions biologiques permettent de disposer d'une base solide pour la poursuite de l'objectif. Des difficultés subsistent toutefois pour évaluer les résultats de ces programmes en raison d'un manque d'outils adaptés. En effet, le programme de restauration des poissons migrateurs de la Dordogne repose encore sur des schémas techniques qui ont été définis au démarrage des opérations et qui ne prévoyaient pas, à cette époque, les outils de suivi et d'évaluation suffisants pour apprécier la situation actuelle. Ces constats interviennent dans un changement général du contexte de gestion des cours d'eau et des milieux naturels aquatiques (décentralisation, loi sur l'eau de 1992), et dans le cadre d'une évolution importante de l'implication de certains partenaires techniques et financiers dans les programmes.

Ces éléments ont amené l'Etablissement Public Interdépartemental DORdogne (E.P.I.DOR.), qui regroupe les collectivités territoriales concernées par le bassin versant de la Dordogne, à animer une réflexion destinée à actualiser les stratégies de gestion et à renforcer la prise en compte de la restauration des poissons migrateurs dans les projets politiques de gestion intégrée du bassin de la Dordogne. Cette réflexion, menée à l'échelle du bassin versant de la Dordogne, a fait appel à l'implication collective de multiples intervenants, pour définir un projet de gestion commun à l'ensemble des espèces migratrices présentes sur le territoire.

Une stratégie intitulée Objectif Retour aux Sources (GUERRI et PUSTELNIK, 1998) est aujourd'hui la base d'éventuels engagements contractuels entre les Collectivités, l'Etat, l'Agence de l'Eau, l'Europe et les acteurs socio-économiques du bassin.

Mots-clés : bassin versant, Dordogne, gestion intégrée, poissons migrateurs, 


\title{
THE METHODOLOGICAL BASES FOR A MIGRATORY FISH MANAGEMENT PROJECT IN THE DORDOGNE BASIN.
}

\begin{abstract}
Operations aimed at the restoration of migratory fish in the Dordogne basin began in 1978. Some 20 years later, much has been achieved in the way of knowledge gained, new legislation, and physical and biological developments. This provides a solid foundation for the achievement of the original goal. Evaluating the results of these programmes still presents difficulties, however, because of a lack of suitable means. The project for restoring migratory fish in the Dordogne, in fact, still lays on technical schemes which were defined at the outset of the operations and which did not foresee, at the time, adequate evaluation tools for appraising the present situation. These facts intervene at a period of general context change for the management of water systems and aquatic environment (for instance, decentralisation and new water laws), in which certain technical and financial partners have changed their involvement in the programmes.
\end{abstract}

Such elements have led the Etablissement Public Interdépartemental DORdogne, officially-backed body known as E.P.I.DOR., which embraces the territorial groups and communities affected by the Dordogne basin, to involve itself in considering ways to management strategies and to reinforce the point that the restoration of migratory fish must be taken into account in the political projects for an integrated development of the river system. This reasoning has been managed at the entire Dordogne basin scale and called on the collective involvement of numerous participants to integrate every species of migratory fish in one and the same management project.

A strategy named Objectif Retour aux Sources (GUERRI et PUSTELNIK, 1998) now serves as a basis for the eventual preparation of contractual undertakings between the communal groups, the State, the Adour-Garonne water agency, Europe, and all in the Dordogne basin who figure under a socio-economic heading.

Key-words : hydrographical basin, Dordogne, integrated management, migratory fish, strategy.

\section{INTRODUCTION}

Les premières opérations coordonnées sur les poissons migrateurs ont démarré, sur le bassin de la Dordogne, avec le lancement du « plan saumon " initié en 1975 et dirigé, au départ, par le Ministère de l'Environnement (ROGUET, 1993). Avant cette date, et depuis le début du siècle, quelques actions ponctuelles ont été menées pour tenter par exemple de résoudre le problème du franchissement des barrages sur la Dordogne, ou pour adapter la réglementation de la pêche à la disparition de l'esturgeon. Ces actions sont toutefois restées isolées et n'ont jamais permis d'aboutir à des résultats satisfaisants (PUSTELNIK, 1982 ; ROGUET, 1983).

Le plan saumon s'est rapidement organisé et développé, intégrant progressivement les autres espèces présentes sur le bassin (ANONYME, 1981), notamment dans le cadre du Contrat Retour aux Sources du Ministère de l'Environnement et du Conseil Supérieur de la Pêche (PORCHER, 1992). Aujourd'hui, le bilan des actions réalisées met en avant d'importants acquis en matière de connaissances sur les espèces et les cours d'eau, de réglementation (habitats et pêche), d'aménagements (dispositifs de franchissement, stations de contrôle) et de reconstitution de stock (structures et techniques de repeuplement en saumons) (BOYER et al., in press). Ces réalisations forment désormais une base solide pour la poursuite de l'objectif migrateur sur le bassin de la Dordogne. 
On peut considérer que l'essentiel des opérations de restauration est accompli, avec les investissements les plus lourds. Cette appréciation correspond au fait que l'on dispose maintenant de la base permettant de passer d'un processus de restauration à un processus de gestion (DULUDE, 1995). Ces acquis ne sont obtenus que depuis peu de temps : à l'exemple du saumon, libre circulation et repeuplements significatifs ne sont réalisés que depuis moins de dix ans (DULUDE et al., 1997), ce qui représente deux ou trois générations, ce qui est très peu à l'échelle des processus génétiques d'acclimatation.

Depuis quelques années on constate également une importante évolution du contexte social dans lequel s'inscrivent les opérations migrateurs, avec l'émergence de nouvelles perceptions et d'une certaine appropriation de la part des acteurs du bassin. Ceci s'observe dans le domaine des décisions politiques où la restauration des poissons migrateurs figure souvent parmi les axes prioritaires des programmes de gestion des cours d'eau comme par exemple les contrats de rivière, dans les procédures administratives dont certaines concernent les poissons migrateurs de façon ciblée (arrêtés de protection du biotope), et même dans des perspectives de développement économique, à travers lexploitation halieutique ou l'image de marque pour le développement touristique.

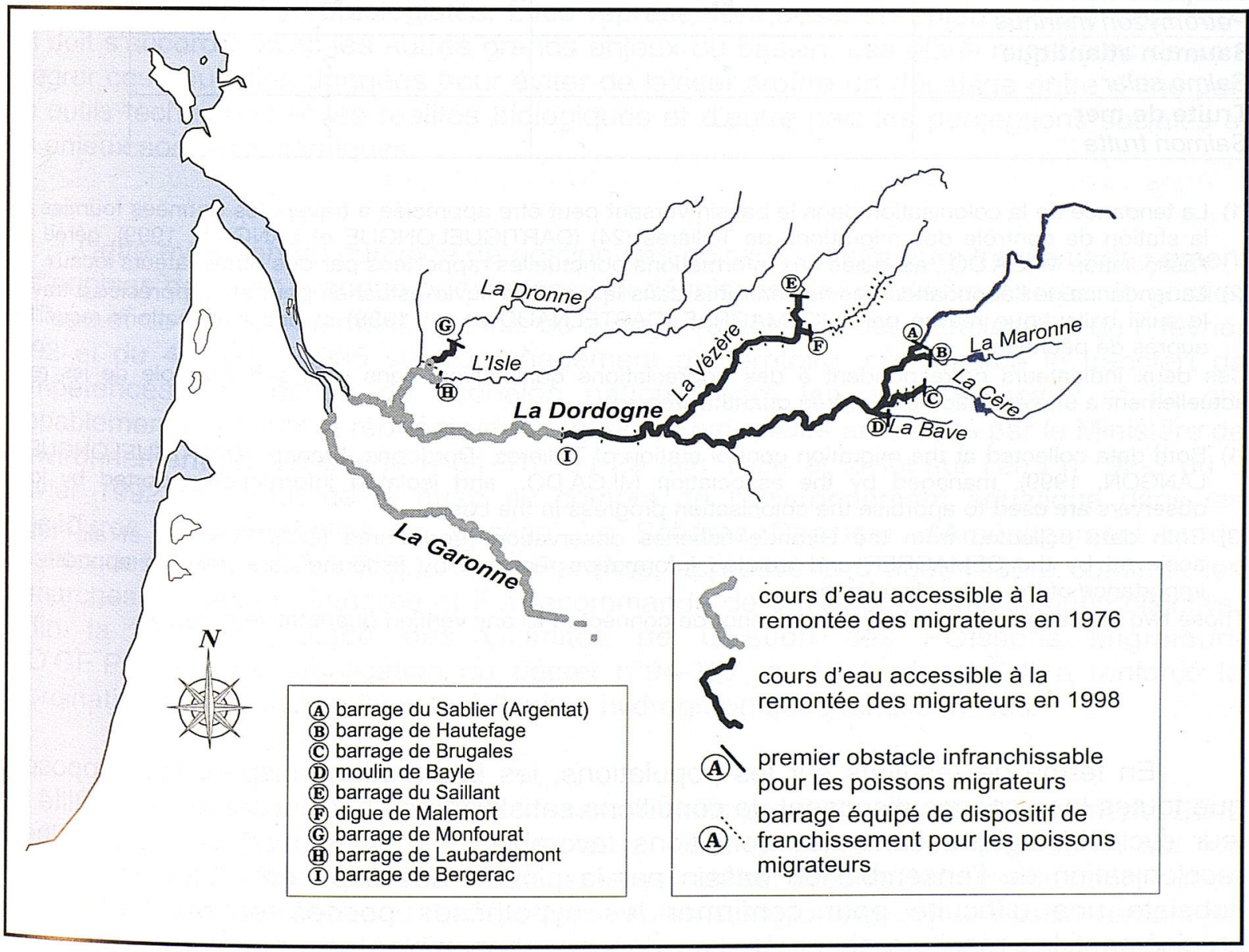

Figure1

Ełat de l'accessibilité du bassin versant de la Dordogne en 1976 et en 1998.

Figure1

Fish circulation status of the Dordogne system in 1976 and 1998. 


\section{Tableau I}

Les éléments disponibles pour les gestionnaires sur la tendance de l'évolution des populations de poissons migrateurs sur le bassin de la Dordogne.

\section{Table I}

\section{Available information for managers to appraise the evolution of migratory fish populations in the Dordogne system.}

\begin{tabular}{|c|c|c|}
\hline Espèce & $\begin{array}{c}\text { Indice de colonisation (1) } \\
\text { bassin versant }\end{array}$ & $\begin{array}{l}\text { Indicateur halieutique (2) } \\
\text { estuaire }\end{array}$ \\
\hline $\begin{array}{l}\text { Esturgeon } \\
\text { Acipenser sturio }\end{array}$ & + & $?$ \\
\hline $\begin{array}{l}\text { Grande alose } \\
\text { Alosa alosa }\end{array}$ & 7 & $\leftrightarrow \mathrm{a} r$ \\
\hline $\begin{array}{l}\text { Alose feinte } \\
\text { Alosa fallax }\end{array}$ & $?$ & $?$ \\
\hline $\begin{array}{l}\text { Anguille européenne } \\
\text { Anguilla anguilla }\end{array}$ & $\neq$ & $\mapsto$ \\
\hline $\begin{array}{l}\text { Lamproie fluviatile } \\
\text { Lampetra fluviatilis }\end{array}$ & $?$ & $\notin ?$ \\
\hline $\begin{array}{l}\text { Lamproie marine } \\
\text { Petromyzon marinus }\end{array}$ & $\rightarrow$ & $\rightarrow$ \\
\hline $\begin{array}{l}\text { Saumon atlantique } \\
\text { Salmo salar }\end{array}$ & $r$ & $?$ \\
\hline $\begin{array}{l}\text { Truite de mer } \\
\text { Salmon trutta }\end{array}$ & $\rightarrow$ & $?$ \\
\hline
\end{tabular}

(1) La tendance de la colonisation dans le bassin versant peut être appréciée à travers les données fournies par la station de contrôle des migrations de Tuilières (24) (DARTIGUELONGUE et LANGON, 1999), gérée par l'association MI.GA.DO, associée aux informations ponctuelles rapportées par des observateurs locaux.

(2) La tendance de l'abondance des flux entrants dans le système fluvio-estuarien peut être appréciée à travers le suivi halieutique réalisé par le CEMAGREF (CASTELNAUD et al., 1998) et des informations recueillies auprès de pêcheurs.

Ces deux indicateurs correspondent à des appréciations qualitatives, sans qu'il soit possible de les relier actuellement à une évolution démontrée quantitativement.

(1) Both data collected at the migration control station of Tullières -Dordogne, France- (DARTIGUELONGUE et LANGON, 1999), managed by the association MI.GA.DO, and isolated information reported by local observers are used to appraise the colonisation progress in the basin.

(2) Both data collected from the Gironde fisheries observation programme (CASTELNAUD et al., 1998), achieved by the CEMAGREF, and isolated information reported by fishermen are used to appraise the importance of the upwards migration.

Those two indicators are qualitative and cannot be connected to any verified quantitative evolution.

En terme de résultats sur les populations, les informations disponibles supposent que toutes les espèces disposent de conditions satisfaisantes pour accomplir la totalité de leur cycle biologique dans des conditions favorables. On a en effet pu assister à la recolonisation de l'ensemble du bassin par la plupart des espèces (Figure 1), mais il subsiste une difficulté pour confirmer les hypothèses posées et pour interpréter précisément les résultats observés. Les données disponibles ne permettent en effet de tirer que de grandes tendances, et ce pour certaines espèces seulement (Tableau I). Cette situation résulte du fait que la plupart des outils actuels reposent encore sur des schémas techniques qui ont peu évolué depuis le lancement des opérations en 1975. Elle a pour conséquence une grande difficulté d'obtenir les informations objectives à même de répondre aux nouvelles données sociales qui émergent sur le bassin versant, avec parfoils d'importants décalages par rapport aux réalités biologiques. 


\section{LE CONTEXTE DE GESTION}

Ces constats, et notamment l'émergence d'une appropriation sociale, permettent d'imaginer les opérations migrateurs, non plus comme un projet exclusivement technique, mais comme l'une des composantes d'un contexte de gestion intégrée. Cette approche apparaît désormais comme une nécessité pour progresser plus avant. Les blocages rencontrés actuellement ont presque toujours leur origine dans un conflit d'usage ou une divergence d'intérêt, et les arguments techniques disponibles ne suffisent pas à les résoudre. II en est par exemple ainsi de désaccords entre pêcheurs et administrations sur la réalité de l'impact d'un mode de pêche sur la ressource, de différends entre propriétaires et gestionnaires sur l'appréciation de la qualité d'obstacle d'un ouvrage hydraulique ou encore de mésententes entre protecteurs de la nature et exploitants hydroélectriques sur les conséquences des marnages dus au fonctionnement des grands barrages. Cette approche correspond également à une réalité car les poissons migrateurs sont de plus en plus considérés comme bases d'un développement, au travers de l'image de qualité des rivières qu'ils représentent, dans un bassin versant où plus de la moitié des ressources économiques provient du tourisme.

Les questions relatives aux poissons migrateurs ne sont donc plus le seul fait des techniciens et des hydrobiologistes. Elles représentent aussi un enjeu socio-économique qui doit s'accorder avec les autres grands enjeux du bassin. Les plans migrateurs devront intégrer ces nouvelles données pour éviter de laisser croître un décalage entre d'une part les outils techniques et les réalités biologiques et d'autre part les perceptions sociales et les enjeux socio-économiques.

Cette évolution du contexte de gestion des poissons migrateurs trouve certainement ses origines dans les grandes orientations qui définissent les nouveaux cadres de gestion de l'eau et de l'environnement. Les lois du 2 mars 1982 sur la décentralisation, du 6 février 1992 et du 4 février 1995 sur l'aménagement du territoire, ont amorcé le transfert de compétences de gestion de l'échelon national vers les acteurs locaux et ils ont probablement contribué à réorienter les stratégies nationales amorcées par le Ministère de l'Environnement. La loi pêche $n^{\circ} 84-512$ du 29 juin 1984 et la loi sur l'eau $n^{\circ} 92-3$ du 3 janvier 1992 ont renforcé la prise en compte de l'environnement aquatique dans les procédures institutionnelles de gestion. Le Schéma Directeur d'Aménagement et de Gestion des Eaux (S.D.A.G.E.) Adour-Garonne, approuvé le 6 août 1996 a confirmé les démarches de gestion intégrée et il a recommandé de développer l'implication collective. Enfin la mise en place des COmités de GEstion des POissons Mlgrateurs (CO.GE.PO.MI.), par application du décret $n^{\circ} 94-157$ du 16 février 1994, a renforcé la coordination des actions à l'échelle d'unités hydrographiques fonctionnelles.

Ces engagements se sont évidemment traduits par d'importants changements dans limplication et le positionnement des acteurs. L'Etat a reporté son implication sur les services déconcentrés et sur ses établissements publics, avec la difficulté de coordination de 36 administrations (Tableau II) départementales et régionales sur le bassin versant de la Dordogne. Les collectivité territoriales et l'Agence de l'eau sont apparues comme de nouveaux partenaires ne bénéficiant pas de toute l'historique des programmes migrateurs, et devant définir les modalités de leur future intervention. Les scientifiques et les techniciens ont rencontré de plus en plus de difficultés à faire valoir leurs arguments dans un contexte de gestion en pleine mutation. Les pêcheurs ont dû adapter leur positionnement dans cette nouvelle démarche de gestion collective, en élargissant leurs partenariats. 


\section{Tableau II}

La multiplicité des services déconcentrés de l'Etat impliqués dans les opérations de gestion des poissons migrateurs.

\section{Table II}

\section{Abundance of local state authorities involved in management of migratory fish.}

\begin{tabular}{|c|c|c|c|}
\hline Service & $\begin{array}{c}\text { Nombre de services } \\
\text { concernés sur le } \\
\text { bassin de la } \\
\text { Dordogne * }\end{array}$ & $\begin{array}{l}\text { Echelle territoriale } \\
\text { de compétence }\end{array}$ & Rôle et Missions \\
\hline $\begin{array}{l}\text { DIREN } \\
\text { Direction regionale de } \\
\text { l"environnement }\end{array}$ & 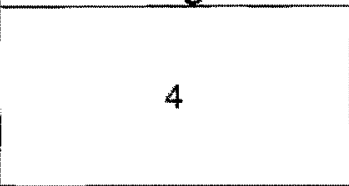 & Régionale & $\begin{array}{l}\text { Gestion et restauration } \\
\text { des populations } \\
\text { migrateurs } \\
\text { Protection des espèces } \\
\text { animales ef végétales el } \\
\text { des milieux naturels }\end{array}$ \\
\hline $\begin{array}{l}\text { DRIRE } \\
\text { Direction regionale } \\
\text { industrie recherche } \\
\text { environnement }\end{array}$ & 4 & Régionale & $\begin{array}{l}\text { Police de l'Environnement } \\
\text { ef des installations } \\
\text { classées }\end{array}$ \\
\hline $\begin{array}{l}\text { MISE } \\
\text { Mission interservice de } \\
\text { l'eau }\end{array}$ & 5 & Départementale & $\begin{array}{l}\text { Protection de la ressource, } \\
\text { préservation des } \\
\text { écosystèmes aquatiques }\end{array}$ \\
\hline $\begin{array}{l}\text { DDAF } \\
\text { Direction departementale } \\
\text { de lagriculture et de la } \\
\text { foret }\end{array}$ & 5 & Départementale & $\begin{array}{l}\text { Gestion et protection des } \\
\text { ressources et des milieux } \\
\text { aquatiques, police des } \\
\text { eaux, police de lla pêche } \\
\text { Police de l'Eau sur le } \\
\text { Domaine prive et police de } \\
\text { la peche }\end{array}$ \\
\hline $\begin{array}{l}\text { DDE } \\
\text { Diroction departementale } \\
\text { de l'équipement }\end{array}$ & 5 & Départementale & $\begin{array}{l}\text { Mise en cewve d'actions } \\
\text { d'amenagement, police de } \\
\text { l'Eau sur le Domaine } \\
\text { public fluvial }\end{array}$ \\
\hline $\begin{array}{l}\text { SMN } \\
\text { Service maritime et de } \\
\text { navigation }\end{array}$ & 1 & Départementale & $\begin{array}{l}\text { Mise en puwre d'actions } \\
\text { d'amenagement, police de } \\
\text { 'Eau sur le Dormaine } \\
\text { putblic fluwial }\end{array}$ \\
\hline $\begin{array}{l}\text { DRAM } \\
\text { Direction, regionale des } \\
\text { affaires martimes }\end{array}$ & 2 & Régionale & Gestion des popullations \\
\hline $\begin{array}{l}\text { DDAW } \\
\text { Direction departementale } \\
\text { des affaires maritimes }\end{array}$ & 2 & Départementale & Police de la peoche \\
\hline $\begin{array}{l}\text { CSP - DR } \\
\text { Délagetions régionales du } \\
\text { conseil superrieur de la } \\
\text { peche }\end{array}$ & 3 & Régionale & $\begin{array}{l}\text { Gestion piscicole, } \\
\text { ingénierrie }\end{array}$ \\
\hline $\begin{array}{l}\text { CSP - brigades } \\
\text { Brigades départementales } \\
\text { et brigades mobiles } \\
\text { d'interwention du conseil } \\
\text { superieur de la pecthe }\end{array}$ & 6 & Départementale & $\begin{array}{l}\text { Surveillanduce die latache, } \\
\text { appusi technique, conseil, } \\
\text { gestion piscicole }\end{array}$ \\
\hline
\end{tabular}

* Le secteur concerne par les plans migrateurs sur le bassin de la Dordogne implique 5 departements (CharenteMaritime, Gironde, Dordogne, Lot, Correze) et quatre régions (Poitou-Charentes, Aquitaine. Midi-Pyrénées, Limousing.

\section{LES PRINCIPES D'UNE STRATÉGIE DE BASSIN}

L'ensemble de ces éléments a amené l'établissement public E.P.I.DOR., qui regroupe les collectivités territoriales du bassin de la Dordogne, à amorcer une réflexion pour definir les voies dimplication de ses collectivités membres. Lobjectif de cette réflexion était de definir les interventions qui leur permettraient de garantir la meilleure prise en compte possible de la restauration et du développement des poissons migrateurs dans les politiques de gestion intégrée du bassin versant de la Dordogne. Les 
gestionnaires du bassin, au sein notamment du CO.GE.PO.MI., ont souhaité profiter de cette réflexion générale et ils ont décidé de mettre cette contribution à profit pour servir de base à l'actualisation des stratégies de gestion des poissons migrateurs.

\section{Trois grands principes ont guidé l'analyse:}

\section{Un projet intégrant huit espèces migratrices}

Les 8 espèces de grands migrateurs amphihalins ont été abordés sur un pied d'égalité, tous pouvant être liés à un enjeu de gestion. II s'agit de : l'esturgeon européen (Acipenser sturio), véritable emblème de la biodiversité et de la préservation d'un patrimoine naturel européen ; les deux aloses (Alosa alosa et Alosa fallax), ainsi que les deux lamproies fluviatile et marine (Lampetra fluviatilis et Petromyzon marinus) qui sont une ressource importante de la pêche professionnelle et qui représentent des éléments culturels originaux à travers les techniques de pêche et la gastronomie ; l'anguille européenne (Anguilla anguilla), espèce en régression et principale ressource de la pêche professionnelle ; les salmonidés : saumon atlantique (Salmo salar) et truite de mer (Salmo trutta), symbole de qualité des eaux, véritables mythes pour les pêcheurs aux lignes, et à l'origine des plans migrateurs pour ce qui concerne le saumon.

\section{Une approche à l'échelle du bassin versant}

Le second principe de base, évident pour les biologistes, mais beaucoup plus difficile à concrétiser en terme de projet de gestion concerne l'échelle d'intervention : le bassin versant. Idéalement, disposant d'un estuaire commun, la Garonne et la Dordogne auraient du relever d'une réflexion commune. Un contexte probablement plus mûr sur la Dordogne a permis, dans un premier temps, d'engager l'analyse sur l'ensemble du bassin versant Dordogne - estuaire de la Gironde. La déclinaison de cette démarche, basée sur la même méthodologie pourrait prochainement être engagée sur la Garonne, permettant ainsi la coordination des actions entre les deux bassins, en s'appuyant sur des partenariats privilégiés avec les organismes de bassin versant que sont l'association Mlgrateurs GAronne DOrdogne (MI.GA.DO.) et le Syndicat Mixte d'Etudes et d'Aménagement de la Garonne (S.M.E.A.G.).

\section{Une implication collective}

Le troisième principe vise à renforcer la liaison entre aspects techniques et sociaux. II concerne l'implication collective et la participation de tous les acteurs, amenés à intervenir ultérieurement, pour définir le projet global. Cette prise en compte, très en amont, de tous les besoins des partenaires ainsi qu'un retour régulier d'information vers les participants du futur projet, ont été vus comme des moyens de maintenir une bonne mobilisation, de faire partager les connaissances acquises et les contraintes techniques qui structureront les actions à venir, préparant ainsi leur future mise en cuvre (PUSTELNIK et GUERRI, in press). Ce dernier point a nécessité la mise en cuvre de partenariats poussés avec les scientifiques et les techniciens, les pêcheurs, les autres usagers et notamment EDF dont l'implantation sur le bassin de la Dordogne est un élément structurant de la situation du bassin, les administrations et les financiers, les collectivités et les élus.

\section{L'OBJECTIF RETOUR AUX SOURCES}

Le détail de l'analyse est présenté dans un document intitulé Objectif Retour aux Sources, en référence aux Contrats Retour aux Sources qui ont structuré les interventions du Ministère de l'Environnement et du CSP de 1992 à 1999. Ce rapport, actuellement en phase de validation, est une base méthodologique du futur projet actualisé de gestion des 
poissons migrateurs sur le bassin versant de la Dordogne. II présente une analyse détaillée des connaissances disponibles et des problèmes connus pour chacune des écophases des huit espèces de grands migrateurs (GUERRI et PUSTELNIK, 1998). Cette approche permet d'englober la totalité du cycle biologique de chacun des poissons, de situer précisément l'origine des problèmes relevés et d'apprécier leur importance relative à l'examen de facteurs limitants.

L'analyse a été soumise à plusieurs étapes de son avancement, à l'ensemble de ces partenaires. Elle a fait appel aux contributions de plus de 50 personnes, institutions et organismes locaux et nationaux concernés par le thème des poissons migrateurs, avec le double souci de synthétiser les connaissances et de les faire partager à l'ensemble des acteurs du bassin. Les organismes concernés sont mentionnés dans Je Tableau III.

Chaque point présentant un déficit de connaissances ou un problème à résoudre a été classé, pour chaque espèce, selon trois niveaux de priorité. Les 148 points recensés pour les 8 espèces ont ensuite été croisés et regroupés lorsqu'ils relevaient d'une problématique et d'une aire géographique commune, pour aboutir à 60 propositions d'action, à mener sur le court, moyen et long terme (Figure 2).

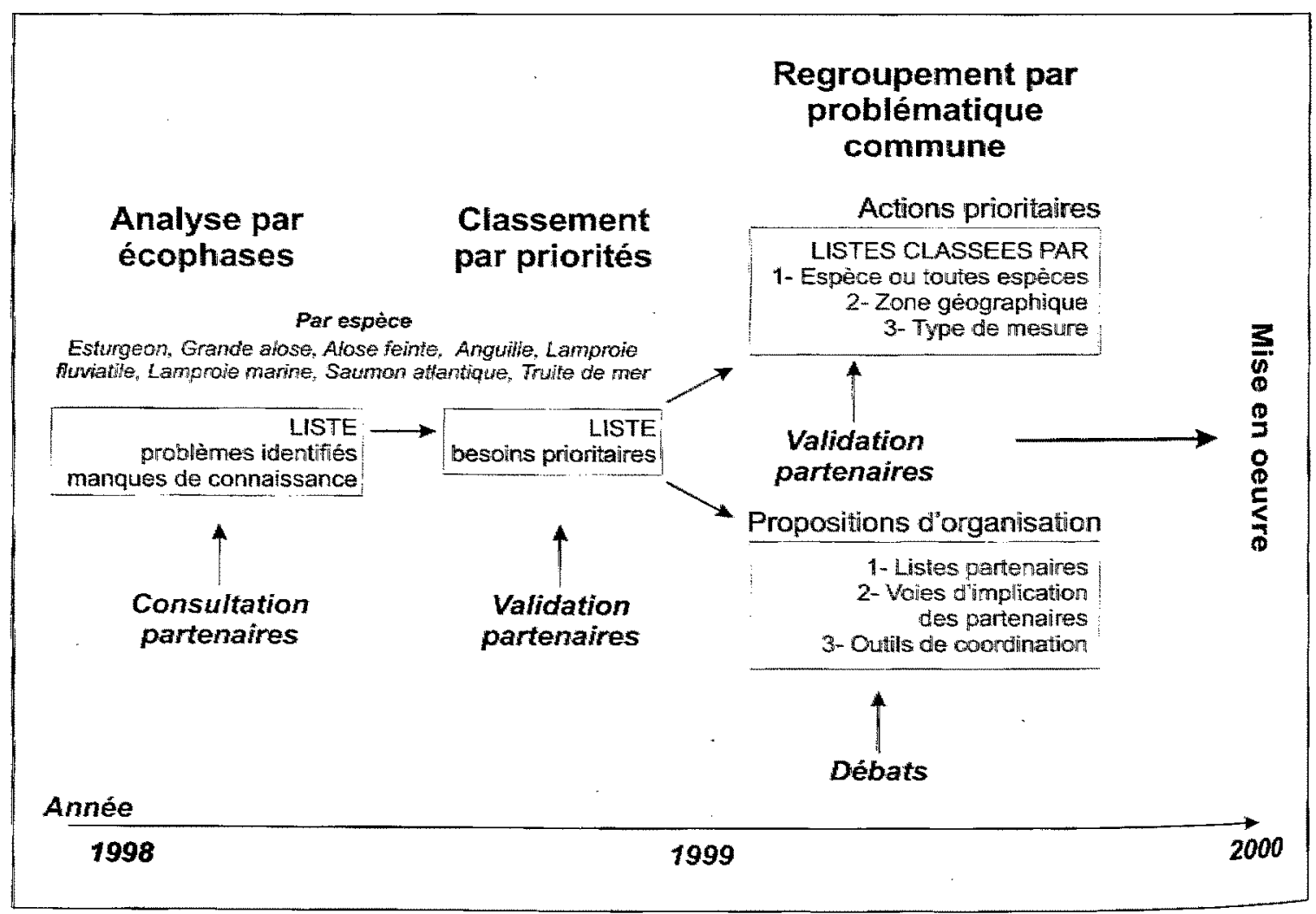

\section{Figure 2}

Synoptic scheme of the analysis achieved in « Objectif Retour aux Sources". 
Tableau III
Liste des
Sources".

Table III

List of the partners involved in the proceeding « Objectif Retour aux Sources ".

\begin{tabular}{|c|c|c|c|c|c|c|c|c|c|}
\hline & & \multicolumn{8}{|c|}{ Rôles et missions } \\
\hline ACTEURS & $\begin{array}{c}\text { Compétence } \\
\text { territoriale }\end{array}$ & \begin{tabular}{|c|}
$\begin{array}{c}\text { Décisio- } \\
\text { nel }\end{array}$ \\
\end{tabular} & \begin{tabular}{|c|}
$\begin{array}{c}\text { Réglem- } \\
\text { entaire }\end{array}$ \\
\end{tabular} & $\begin{array}{c}\text { Admini- } \\
\text { stratif }\end{array}$ & $\begin{array}{c}\text { Finan- } \\
\text { cier }\end{array}$ & $\begin{array}{c}\text { Scienti- } \\
\text { fique }\end{array}$ & \begin{tabular}{c|}
$\begin{array}{c}\text { Techni- } \\
\text { que }\end{array}$ \\
\end{tabular} & $\begin{array}{l}\text { Commur } \\
\text { nication }\end{array}$ & $\begin{array}{l}\text { Péda- } \\
\text { gogie }\end{array}$ \\
\hline \multicolumn{10}{|c|}{ Acteurs ayant participé à l'élaboration et à la validation de la stratégie Objectif Retour aux Sources } \\
\hline $\begin{array}{l}\text { Ministère Aménagement du } \\
\text { Teritoire ef Environnement }\end{array}$ & National & $\mathrm{x}$ & $x$ & $x$ & $\mathrm{x}$ & & & $x$ & $X$ \\
\hline GHAAPPE & National & & & & & $x$ & $x$ & & \\
\hline EDF Etudes et Recherche & National & & & & & $\mathrm{X}$ & $x$ & & \\
\hline Association T.O.S. & National & $\mathrm{X}$ & & & & & & $x$ & $x$ \\
\hline $\begin{array}{l}\text { Aggence de l'Eau Adour } \\
\text { Garonne }\end{array}$ & Adour Garonne & $x$ & & $x$ & $x$ & & $x$ & $x$ & $x$ \\
\hline CSP DR Toulouse & Adour Garonne & & & $x$ & $x$ & $x$ & $x$ & & \\
\hline $\begin{array}{l}\text { Mission technique poissons } \\
\text { migrateurs }\end{array}$ & Adour Garonne & & & & & & $x$ & & \\
\hline Cemagref & Sud-ouest & & & & & $x$ & $\mathrm{X}$ & & \\
\hline INRA & Sud-ouest & & & & & $x$ & $\mathrm{x}$ & & \\
\hline IFREMER & Sud-ouest (maritime) & & & & & $x$ & $x$ & & \\
\hline ENSAT & Sud-cuest & & & & & $x$ & $x$ & & \\
\hline Association ACVF & Adour Garonne & $x$ & & & & $x$ & $x$ & $x$ & $x$ \\
\hline COGEPOMI & Garonne Dordogne & $x$ & & & & & & $x$ & \\
\hline CSP BMI & Garonne Dordogne & & $x$ & & & & & & \\
\hline ML.GA.DO. & Garonne Dordogne & $x$ & & & & $x$ & $x$ & $x$ & $x$ \\
\hline E.P.IDOR & Dordogne & $x$ & & $\mathrm{X}$ & & $x$ & $x$ & $x$ & $x$ \\
\hline Association AGEDRA & Régional & $x$ & & & & $X$ & $x$ & $x$ & $\mathrm{X}$ \\
\hline DIREN & Régions adm. & & $x$ & $x$ & $x$ & $x$ & $x$ & $x$ & \\
\hline $\begin{array}{l}\text { Conités régionaux pêches } \\
\text { maritimes }\end{array}$ & Régions adm. & $x$ & & & & & & $x$ & \\
\hline EDF Energie Aquitaine & Régions adm. & $x$ & & $x$ & $x$ & & $x$ & & \\
\hline EDF Energie Midi-Pyrénées & Régions adm. & $\mathrm{X}$ & & $x$ & $x$ & & $x$ & & \\
\hline CSP DR Clermont & Régions adm. & & & $x$ & $x$ & $x$ & $x$ & & \\
\hline SEPANSO & Regions adm. & $x$ & & & & & & $x$ & $x$ \\
\hline Conseils Régionaux & Régions adm. & $x$ & & $x$ & $x$ & & & & \\
\hline $\begin{array}{l}\text { A.A.I.P.P.B.G (pêcheurs } \\
\text { fluviaux) }\end{array}$ & Garome Dordogne & $x$ & & $x$ & & & $x$ & $x$ & \\
\hline $\begin{array}{l}\text { A.A.D.P.P.E.D.G (pëcheurs } \\
\text { Thuviaux) }\end{array}$ & Département Gironde & $x$ & & $x$ & & & $x$ & $x$ & \\
\hline ASsociation ADES & Estuaire Gironde & $x$ & & & & & $x$ & $x$ & $x$ \\
\hline M.IS.E & Départements & & $x$ & $x$ & & & & $x$ & \\
\hline DDAM & Départements & & $\mathrm{X}$ & $x$ & & & & $x$ & \\
\hline $\begin{array}{l}\text { CSP brigades } \\
\text { dépatementales }\end{array}$ & Départements & & $x$ & $x$ & & & $x$ & & \\
\hline Féderations de pêche & Départements & $x$ & & $\mathrm{x}$ & $x$ & & $x$ & $x$ & \\
\hline Conseils Généraux & Départements & $x$ & & $X$ & $x$ & & & & \\
\hline EDF centrale du Blayais & Local & $\mathrm{x}$ & & $\mathrm{x}$ & $x$ & & $\mathrm{x}$ & & \\
\hline \multicolumn{10}{|c|}{ Acteurs complémentaires pressentis pour la mise en ceuvre de la stratégie Objectif Retour aux Sources } \\
\hline GPISAM & National & & & & & $x$ & & & \\
\hline Préfecture de bassin & Adour Garonne & $\bar{x}$ & $x$ & $\mathrm{x}$ & & & & & \\
\hline Comite de bassin & Adour Garonne & $\mathrm{X}$ & & & & & & & \\
\hline $\begin{array}{l}\text { Commission Milieux Naturels } \\
\text { Aqquatiques Adour Garonne }\end{array}$ & Adour Garonne & $x$ & & & & & & & \\
\hline Université Toulouse / & Sud-ouest & & & & & $\mathrm{X}$ & & & \\
\hline Université Bordeaux I & Sud-ouest & & & & & $x$ & & & \\
\hline Institut Milieux Aquatiques & Régional & & & & & $x$ & $x$ & $x$ & $x$ \\
\hline $\begin{array}{l}\text { Comités locaux pêches } \\
\text { martimes }\end{array}$ & Quartiers maritimes & $x$ & & $x$ & & & & $x$ & \\
\hline Croupements intercommunaux & Local & $x$ & & $x$ & $x$ & & $x$ & $x$ & \\
\hline A.P.P.M.A, & Local & $x$ & & $x$ & $x$ & & $x$ & $x$ & \\
\hline Riverains & Local & $x$ & & & & & & & \\
\hline Conservatoire de l'estuaire & Local & $x$ & & & & & & $x$ & $x$ \\
\hline Port autonome de Bordeaux & Local & $x$ & & $x$ & $x$ & & $x$ & & \\
\hline
\end{tabular}


Dans une seconde partie, l'Objectif Retour aux Sources présente des propositions pour la mise en œuvre de ces actions, en prenant en compte la contribution des opérations déjà engagées, les moyens et les compétences des acteurs locaux, régionaux et nationaux susceptibles de participer au projet (Tableau III). Cette partie de l'analyse étudie l'implication indispensable ou facultative de chaque partenaire dans les différentes phases de la mise en cuvre de chaque action, c'est-à-dire : décisionnelle, réglementaire, administrative, financière, scientifique, technique, informative et pédagogique.

\section{CONCLUSIONS ET PERSPECTIVES}

Ce rapport doit prochainement aboutir à la mise en place d'une organisation qui favorisera la mise en ceuvre coordonnée d'un projet collectif à l'échelle du bassin versant. La forme envisagée pour cette organisation pourrait s'inspirer du modèle des contrats de rivière qui comprennent un comité de coordination, un animateur de bassin, des programmes d'actions détaillés, ainsi qu'un ensemble d'outils techniques (bases de données, atlas, outils d'échange d'information) ayant pour but de placer tous les partenaires sur un même niveau d'information.

L'Objectif Retour aux Sources sert actuellement de base à la préparation d'accords contractuels entre les partenaires financiers, à travers les outils financiers que sont : les contrats de plan Etat-Région sur lesquels est engagée une démarche interrégionale sur le bassin de la Dordogne, la Politique Territoriale Bassin Dordogne, négociée entre les départements et l'Agence de l'Eau Adour-Garonne ainsi que les programmes européens.

II pourra également préparer la mise en place de chartes techniques ou de conventions de partenariat pour formaliser l'engagement des acteurs du bassin autour d'objectifs communs.

\section{BILBLIOGRAPHIE}

ANONYME, 1981. Plan Poissons migrateurs 1981-1986. Ministère de l'EnvironnementDirection de la Protection de la Nature, $20 \mathrm{p}$.

BOYER S., GUERRI O., PUSTELNIK G., in press. Situation des programmes migrateurs sur l'ensemble des bassins versants Garonne et Dordogne.

CASTELNAUD G., LAMBERT P., ROCHARD E., 1998. Surveillance halieutique de l'estuaire de la Gironde. Suivi statistique 1996, étude de la faune circulante 1997. Etude CEMAGREF - Bordeaux $n^{\circ}$ 97. EDF CPN Blayais, $153 \mathrm{p}$.

DARTIGUELONGUE J., LANGON M., 1999. Contrôle du fonctionnement de l'ascenseur à poissons de Tuilières en 1998. Suivi de l'activité ichtyologique. Rapport MI.GA.DO. G7-99-RT, $26 \mathrm{p}$.

DULUDE P., 1995. Vers une restauration réussie du saumon et des autres poissons migrateurs dans la Dordogne. Conseil Général de la Dordogne, $18 \mathrm{p}$.

DULUDE P., GUERRI O., PUSTELNIK G., CHEVRE P., 1997. Objectif 2003 : propositions pour une stratégie de gestion et de repeuplement du saumon atlantique dans les cours d'eau du bassin de la Dordogne. E.P.I.DOR., MI.GA.DO., 34 p.

GUERRI O., PUSTELNIK G., 1998. Objectif Retour aux Sources, bassin versant Dordogne ; document révisé. E.P.I.DOR., $216 \mathrm{p}$.

PORCHER J.P., 1992. Poissons migrateurs, Contrat « retour aux sources ». Ministère de l'Environnement, Conseil Supérieur de la Pêche, 169 p. 
PUSTELNIK G., 1982. Les saumons de la rivière Dordogne - 1189-1981. Ministère de l'Environnement, Fédération départementale de la pêche de la Dordogne, $54 \mathrm{p}$.

PUSTELNIK G., GUERRI O., in press. Analysis of partnership and conservation requirements for a threatened species, (Acipenser sturio L.) : towards the implementation of a recovery plan. Communication International symposium on conservation of Acipenser sturio. Madrid, Sevilla, 6-11 sept 1999.

ROGUET M., 1983. Bilan et perspectives du plan saumon. Le retour du saumon. AdourGaronne, $n^{\circ} 26$, juin 1983, 3-9.

ROGUET M., 1993. Bilan du programme de restauration des poissons migrateurs dans le bassin Adour-Garonne). Actes du colloque Pour un retour des poissons migrateurs, Adour, Charente, Dordogne, Garonne. Agence de l'Eau Adour-Garonne (1993), $191 \mathrm{p}$. 